\title{
О ПОСТРОЕНИИ СПЕЦИАЛЬНОЙ ТЕОРИИ ОТНОСИТЕЛЬНОСТИ
}

\section{Введение}

В статье [ $\left.{ }^{1}\right]$ показано, что при построении специальной теории относительности можно полностью обойтись без постулата постоянства скорости света и без замены его требованием групповости инерциальных преобразований. Достаточно исходить только из принципа относительности. В результате некоторого мысленного опыта приходим или к классической теории, или к теории относительности, причем во втором случае в теорию входит универсальная скорость, которую можно отождествить со скоростью света. Этот метод представлен в двух варнантах. В одном из них используются наряду с принципом относительности законы сохранения массы и импульса, в другом они не нужны, а все результаты получаются из сравнения длин движущихся стержней.

Таким образом, для построения специальной теории относительности требуется в основном лишь отказ от классической теории при сохранении принципа относительности. Отметим, что отказ от іклассической теории не является слишком сильным требованием, поскольку он необходим и в случае замены второго постулата Әйнштейна групповым постулатом (см. $\left[{ }^{2-5}\right]$ или, из новой литературы, $\left.\left[{ }^{6}\right]\right)$. Поэтому наш метод өкономнее методов, основанных на групповом постулате.

В настоящей статье изложим тот же метод в ином виде и тоже в двух вариантах. В некотором смысле предлагаемый здесь новый способ еще проще и экономнее.

\section{Вывод основного функционального уравнения}

В первом варианте метода используются законы сохранения массы и импульса. Рассмотрим распад покоящейся частицы с массой покоя $M$ на две одинаковые частицы с массой покоя $m$. Их скорости, в силу сохранения импульса, равны и противоположны, а в силу сохранения массы

$$
M=2 m \gamma(v),
$$

где $v$ - скорость вторичных частиц, а $\gamma(v)$ - множитель, учитывающий предполагаемую зависимость массы от скорости. Перейдем в систему покоя одной из вторичных частиц. Скорость первичной частицы будет там $v$, а скорость другой вторичной обозначим через $u$. Сохранение массы и импульса запишется в виде:

$$
\begin{aligned}
& M \gamma(v)=m+m \gamma(u), \\
& M v \gamma(v)=m u \gamma(u) .
\end{aligned}
$$


Исключая из уравнений (1)-(3) массы $M$ и $m$, находим

$$
2 \gamma(v)=\gamma^{-1}(v)(1+\gamma(u))=v^{-1} \gamma^{-1}(v) u \gamma(u) .
$$

Отсюда выражаем $\gamma(u)$ и $u$ :

$$
\begin{gathered}
\gamma(u)=2 \gamma^{2}(v)-1, \\
u=2 v \gamma^{2}(v)\left[2 \gamma^{2}(v)-1\right]^{-1} .
\end{gathered}
$$

Следовательно,

$$
2 \gamma^{2}(v)-1=\gamma\left(\frac{2 v \gamma^{2}(v)}{2 \gamma^{2}(v)-1}\right)
$$

Это - функциональное уравнение, определяющее функцию $\gamma(v)$. Чтобы упростить его, введем вместо $\gamma(v)$ функцию

$$
\Gamma(v)=v^{-2}\left(1-\gamma^{-2}(v)\right) .
$$

Тогда уравнение (7) примет вид

$$
\Gamma(v)=\Gamma\left(\frac{2 v}{1+v^{2} \Gamma(v)}\right) .
$$

В самом деле, возводя уравнение (7) в степень -2 , вычитая из единицы и деля на $4 v^{2} \gamma^{4}(v)\left[2 \gamma^{2}(v)-1\right]^{-2}$, находим в левой части $v^{-2}\left(1-\gamma^{-2}(v)\right)$, что равно $\Gamma(v)$, а в правой части $\Gamma\left(\frac{2 v \gamma^{2}(v)}{2 \gamma^{2}(v)-1}\right)$, что равно правой части уравнения (9).

Такого же вида уравнение получается и в другом варианте метода. Пусть стержень длины покоя $l_{1}$ покоится (будем называть его дальше «коротким»), а два других стержня длины покоя $l_{2}$ каждый движутся мимо него с равными и противоположными скоростями $\pm v$. Эти стержни (будем называть их «длинными») имеют, очевидно, и в движении равные длины $l_{2} g(v)$, где $g(v)$ - предполагаемый множитель, определяющий длину движущегося продольно стержня в зависимости от его скорости. Рассмотрим следующие три совпадения концов движущихся стержней (во временной последовательности): 1) совпадение их передних кконцов; 2) совпадение переднего конца каждого из них с задним концом другого; 3 ) совпадение задних концов. Подберем длины $l_{1}$ и $l_{2}$ так, чтобы первое и третье совпадения совершались у правого конца покоящегося стрежня, а совпадение левых концов движущихся стержней - у левого конца покоящегося. Относительное расположение всех трех стержней в эти три момента показано на рис. 1 .

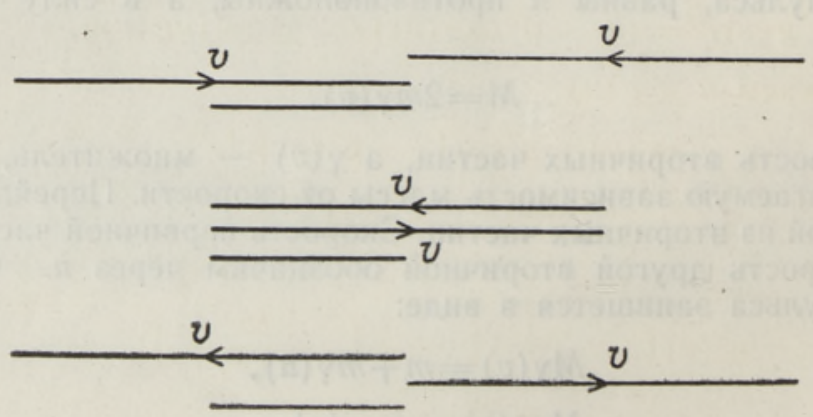

Рис. 1. 
Очевидно, промежуток времени между первым и вторым совпадениями равен промежутку времени между вторым и третьим совпадениями. Расстояние, проходимое одним из длинных стержней за один из этих промежутков времени, равно $l_{1}$, а расстояние, проходимое другим длинным за тот же промежуток времени, равно $l_{2} g(v)-l_{1}$. А так как скорости стержней равны, то $l_{1}=l_{2} g(v)-l_{1}$, т. е.

$$
2 l_{1}=l_{2} g(v) \text {. }
$$

Перейдем в систему покоя одного из длинных стержней, например, движущегося слева направо. Скорость короткого будет тогда равна $v$, а скорость другого длинного обозначим через $u$. Так как длины $l_{2} l_{2} g(u)$ длинных стержней теперь неравны (если $g(u) \neq 1$ ), то в момент совпадения их левых концов правые не совпадают. На рис. 2 показано вза-

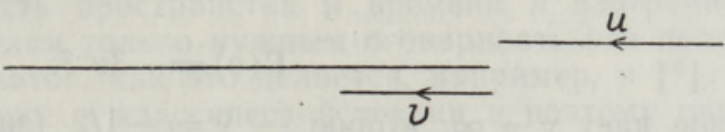

Рис. 2.
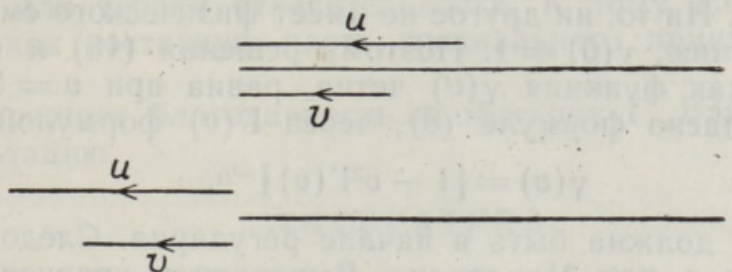

имное расположение стержней при тех же трех совпадениях, что рассмотрены выше: сначала левый конец движущегося длинного стержня совпадает с правыми концами остальных двух стержней; потом совпадают левые концы всех трех стержней; наконец, левый конец неподвижного длинного стержня совпадает с правыми концами остальных двух стержней. Расстояния, проходимые коротким и длинным стержнями между первым и вторым совпадениями, равны $l_{2}-l_{1} g(v)$ и $l_{2}$ соответственно, а расстояния, проходимые ими между вторым и третьим совпадениями, равны $l_{1} g(v)$ и $l_{2} g(u)$. В обоих случаях расстояния пропорциональны скоростям стержней, т. е.

$$
\left(l_{2}-l_{1} g(v)\right) / l_{2}=v / u
$$

и

$$
l_{1} g(v) /\left(l_{2} g(u)\right)=v / u .
$$

Исключим теперь из уравнений $(10)-(12)$ длины $l_{1}$ и $l_{2}$; в результате получим

$$
g(v) / 2=(u-v) /(u g(v))=v g(u) /(u g(v)) .
$$

Отсюда выражаем $u$ и $g(u)$ :

$$
\begin{gathered}
g(u)=g^{2}(v)\left[2-g^{2}(v)\right]^{-1}, \\
u=2 v\left[2-g^{2}(v)\right]^{-1} .
\end{gathered}
$$

Следовательно,

$$
g^{2}(v)\left[2-g^{2}(v)\right]^{-1}=g\left(\frac{2 v}{2-g^{2}(v)}\right) .
$$


Это уравнение эквивалентно уравнению (7) в том смысле, что оба переходят друг в друга при замене $g(v) \rightleftarrows \gamma^{-1}(v)$. Следовательно, величины $\gamma(v)$ и $g(v)$ в любом случае должны удовлетворять соотношению

$$
\gamma(v) g(v)=1 \text {. }
$$

Все выводы в обоих вариантах будут, очевидно, одни и те же. Поэтому вернемся к уравнению (9).

\section{Решение функционального уравнения}

Функциональное уравнение (9), как лепко убедиться, имеет решения

$$
\Gamma(v)=v^{-2}
$$

и

$$
\Gamma(v)=-3 v^{-2} \text {. }
$$

Первое дает $\gamma \rightarrow \infty$, второе $-\gamma=-1 / 2$ (знак минус вытекает здесь из формулы (5)). Ни то, ни другое не имеет физического смысла, и притом, по определению, $\gamma(0)=1$. Поэтому решения (18) и (19) следует отбросить. Так как функция $\gamma(v)$ четна, равна при $v=0$ единице и выражается, согласно формуле (8), через Г(v) формулой

$$
\gamma(v)=\left[1-v^{2} \Gamma(v)\right]^{-1 / 2},
$$

то функция Г(v) должна быть в начале регулярна. Следовательно, ее можно разложить в ряд Маклорена. Вычисляя в уравнении (9) производную по $v$, получаем:

$$
\Gamma^{\prime}(v)=\frac{2\left(1-v^{2} \Gamma(v)-v^{3} \Gamma^{\prime}(v)\right)}{\left(1+v^{2} \Gamma(v)\right)^{2}} \cdot \Gamma^{\prime}\left(\frac{2 v}{1+v^{2} \Gamma(v)}\right),
$$

где штрих означает производную по аргументу. Положив $v=0$, имеем $\Gamma^{\prime}(0)=2 \Gamma^{\prime}(0)$, откуда

$$
\Gamma^{\prime}(0)=0
$$

Беря в (21) вторую производную, полагая $v=0$ и учитывая (22), находим $\Gamma^{\prime \prime}(0)=4 \Gamma^{\prime \prime}(0)$, т. е.

$$
\Gamma^{\prime \prime}(0)=0 \text {. }
$$

Продолжая ту же процедуру и дальше, лепко убеждаемся, что все производные обращаются в нуль. Следовательно,

$$
\Gamma(v)=\text { const. }
$$

Из формулы (20) вытекает, что величина Г должна иметь размерность обратного квадрата скорости; следовательно, если только $\Gamma \neq 0$,

$$
\Gamma=c^{-2},
$$

где $c-$ универсальная скорость, отождествляемая со скоростью света. О невозможности $Г<0$ см. в [ [ $\left.{ }^{1}\right]$. Итак,

и

$$
\gamma(v)=\left(1-v^{2} / c^{2}\right)^{-1 / 2}
$$

$$
g(v)=\left(1-v^{2} / c^{2}\right)^{1 / 2} .
$$

Дальнейшее построение теории относительности известно. 


\section{Заключение}

Ненужность группового постулата как замены второго постулата Эйнштейна демонстрируется в вышеизложенном методе яснее всего тем обстоятельством, что в обоих вариантах метода вводятся в рассмотрение только две инерциальные системы. Правда, способ, изложенный в статье [ [1], тоже не нуждается в групповом постулате, но там используются три инерциальные системы; поэтому метод чист только в силу отсутствия повторных преобразований какой-либо величины из одной инерциальной системы в другую, а оттуда в третью. Новый же метод чист автоматически, так как в нем повторные преобразования вообще никак участвовать не могут.

Отметим еще в заключение, что мы, как обыцн, предполагаем в нашем методе однородность пространства и времени и изотропность пространства. Мы не считаем только нужным оговаривать эти положения в виде особых постулатов, как это делается, например, в [ $\left.{ }^{6}\right]$. Эти положения справедливы уже в классической теории и поэтому они не специфичны для теории относительности. К тому же их можно рассматривать как составную часть специального принципа относительности.

Автор выражает благодарность профессору Г. Вайникко за полезную консультацию.

Л И Т Е Р А У Р А

1. К а рд П., Изв. АН ЭССР, Физ. Матем., 25, 227 (1976).

2. I g n a tow sky, W., Phys. Z., 11, 972 (1910).

3. I g n a tow sky, W., Phys. Z., 12, 779 (1911).

4. Fra nk, Ph., Rothe, H., Phys. Z., 13, 750 (1912).

5. Fr a n k, Ph., R o the, H., Ann. Phys., 34, 825 (1911).

6. Lé v y - Le blond, J.-M., Amer. J. Phys., 44, 271 (1976).

Тартуский государственный университет
Поступила в редакцию 10/III 1977

\section{P. KARD}

\section{ERIRELATIIVSUSTEOORIA OLESEHITAMISEST}

Esitatakse kahes variandis meetod erirelatiivsusteooria ülesehitamiseks erirelatiivsus. printsiibi alusel ilma Einsteini teise postulaadita ja rühmapostulaadita. Esimeses variandis rakendatakse massi ja impulsi jäävuse seadusi osakese lagunemisele kaheks ühesuguseks sekundaarseks osakeseks, eeldades massi võimalikku (teguri $\gamma(v)$ kaudu) söltuvust kiirusest $v$. Teises variandis võrreldakse kolme üksteise suhtes liikuva varda kahe ühepikkuse ja ühe lühema - pikkusi, eeldades pikkuse võimalikku (teguri $g(v)$ kaudu) sōltuvust kijrusest. Tulemusena saadakse seos (17) ja võrrand (9), kus $\Gamma(v)$ on defineeritud valemiga (8). Näidatakse, et vōrrandi (9) ainus algtingimust $\gamma(0)=1$ rahuldav lahend on $\Gamma=$ const. Kui $\Gamma \neq 0$, siis $\Gamma=c^{-2}$, kus $c$ on valguse kiirus. Meetod sarnaneb artiklis [ $\left.{ }^{1}\right]$ esitatud meetodiga, kuid on sellest põhimõtteliselt lihtsam. 


\section{P. KARD}

\section{ZUM AUFBAU DER SPEZIELLEN RELATIVITÄTSTHEORIE}

Es wird eine neue Methode entwickelt zum Aufbau der speziellen Relativitätstheorie auf Grund des speziellen Relativitätsprinzips ohne zweites Postulat Einsteins und ohne Gruppenpostulat. Es gibt zwei verschiedene Gestaltungen dieser Methode. Die erstere beruht auf den Erhaltungssätzen der Masse und des Impulses, angewandt auf den Zerfall eines Teilchens in zwei gleiche sekundäre Teilchen. Dabei wird angenommen, dass die Masse möglicherweise durch einen Faktor $\gamma(v)$ von der Geschwindigkeit abhängt. In letzterer Gestaltung vergleicht man die Längen dreier sich relativ zueinander bewegenden Stäbe, von denen ein kürzerer, die beiden anderen aber längere von gleicher Länge sind. Dabei wird ein Faktor $g(v)$ eingeführt, der eine mögliche Abhängigkeit der Länge von der Geschwindigkeit berücksichtigt. Es stellt sich heraus, dass das Produkt der Faktoren $\gamma(v)$ und $g(v)$ stets gleich eins sein muss (s. Formel (17)). Darüber hinaus wird gezeigt, dass die Funktion $\Gamma(v)$, die mit dem Faktor $\gamma(v)$ durch die Beziehung (8) verknüpft ist, die Gleichung (9) befriedigen muss. Die einzige Lösung dieser Gleichung, die der Anfangsbedingung $\gamma(0)=1$ genügt, ist $\Gamma=$ const. Wenn $\Gamma \neq 0$, dann $\Gamma=c^{-2}$, wo $c$ der Lichtgeschwindigkeit gleichgesetzt werden muss. Diese Methode ist der in einem früheren Aufsatz $\left.{ }^{[}{ }^{1}\right]$ dargelegten Methode ähnlich, doch ist sie in prinzipieller Hinsicht etwas einfacher als jene. 